EPJ manuscript No.

(will be inserted by the editor)

\title{
Boundary-induced phase transitions in a space-continuous traffic model with non-unique flow-density relation
}

\author{
Alireza Namazi ${ }^{1,2}$, Nils Eissfeldt ${ }^{2}$, Peter Wagner $^{3}$, and Andreas Schadschneider ${ }^{1}$ \\ 1 Institut für Theoretische Physik, Universität zu Köln, D-50937 Köln, Germany \\ 2 ZAIK - Center for Applied Informatics, Universität zu Köln, D-50931 Köln, Germany \\ ${ }^{3}$ Institute for Transportation Research, German Aerospace Center, D-12489 Berlin, Germany
}

October 22, 2018

\begin{abstract}
The Krauss-model is a stochastic model for traffic flow which is continuous in space. For periodic boundary conditions it is well understood and known to display a non-unique flow-density relation (fundamental diagram) for certain densities. In many applications, however, the behaviour under open boundary conditions plays a crucial role. In contrast to all models investigated so far, the high flow states of the Krauss-model are not metastable, but also stable. Nevertheless we find that the current in open systems obeys an extremal principle introduced for the case of simpler discrete models. The phase diagram of the open system will be completely determined by the fundamental diagram of the periodic system through this principle. In order to allow the investigation of the whole state space of the Krauss-model, appropriate strategies for the injection of cars into the system are needed. Two methods solving this problem are discussed and the boundary-induced phase transitions for both methods are studied. We also suggest a supplementary rule for the extremal principle to account for cases where not all the possible bulk states are generated by the chosen boundary conditions.
\end{abstract}

PACS. 02.50.Ey Stochastic processes; 45.70.Vn Granular models of complex systems, traffic flow; 05.40.-a Fluctuation phenomena, random processes, noise, and Brownian motion

\section{Krauss-model}

The number of vehicles on highways and in cities is increasing each year causing vehicular traffic to suffer more and more from jams. The phenomena related to traffic jams have attracted the attention of physicists and engineers since almost half a century, trying to develop models describing the features of the real traffic. Generally there are two different approaches: microscopic and macroscopic [1, 2, 3]. Whereas in microscopic models different vehicles and their dynamics can be distinguished, in macroscopic models only densities are considered, similar to hydrodynamcis.

However, the approach of a physicist is usually quite different from that of a traffic engineer. One of the current interests of statistical physicists are the so called "nonequilibrium systems". In microscopic vehicular traffic theories, vehicular traffic is treated as a system of interacting particles driven far from equilibrium and offers the possibility to study various fundamental aspects of the dynamics of truly nonequilibrium systems.

Empirical observations show that the average velocity decreases with increasing vehicle density. So the average current (or flow), which is the product of average velocity and density, is a function of the density. The functional relation between current and density is usually called funda- mental diagram. Its generic form can be understood easily. For small densities all vehicles can move with their desired velocity $v_{\max }$ and the current increases monotonously. For large densities the vehicles interact with each other and the average velocity is much smaller than in the free flow regime. This causes a decrease of the current, with a maximum at an intermediate value.

The traffic model introduced in [4,5, 67, called Kraussmodel in the following, is based on an approach by Gipps [7] considering the braking distance of individual cars. Starting from the assumption of safe driving an update scheme can be formulated in the manner of the well-known Nagel-Schreckenberg (NaSch) model [8,9]. In the Kraussmodel - unlike the NaSch model - the state variables, i.e. space and velocity, are chosen to be continuous. To make the model safe, i.e. free of collisions, a safe velocity $v_{\text {safe }}$ for each car is introduced, which is calculated in every timestep taking into account that there is a maximum acceleration and deceleration rate for each car. The vehicles will be updated in parallel corresponding to discrete time dynamics.

The model has been designed to reproduce the empirial findings in traffic jams [10,11, 12]:

1. There is a density regime with non-unique flow-density relation. 


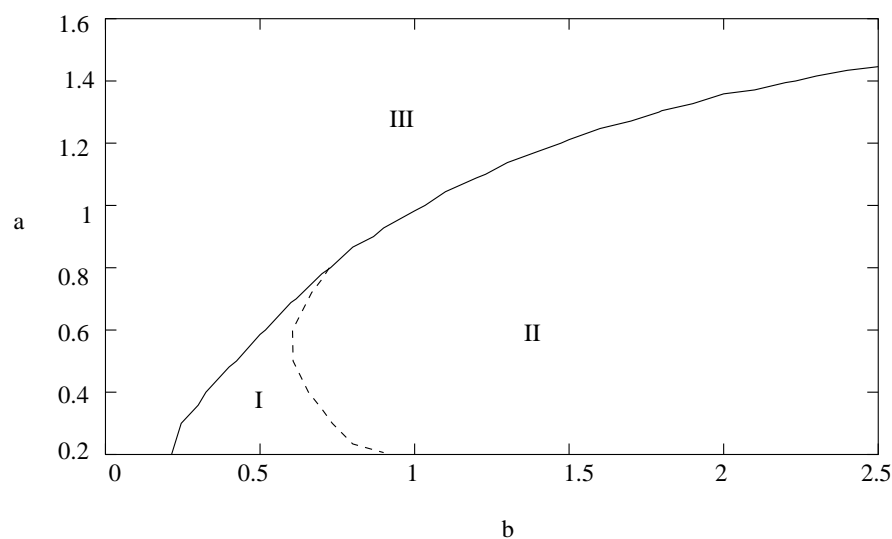

Fig. 1. Classes of qualitatively different behaviour in the Krauss-model. In class $I$, the acceleration $a$ and deceleration $b$ are realistic and all properties $1-4$ of jams are present. In class $I I$ decelerations are large and the properties 1,3 , and 4 of jams are not reproduced. In class $I I I$ accelerations are large and no jams exist (from [5]).

2. Traffic jams can develop and exist under "pure" conditions, i.e. in the absence of any obstacles.

3. The flux out of a jam is not maximal.

4. The outflow from jams is stable.

5. The outflow from jams and the velocity of the downstream front do not depend on the inflow conditions.

These properties are displayed by the model for a certain range of parameters. The model equations proposed in [5] even show a much richer behaviour depending on the relation between the ac- and deceleration capabilities 河, 6. Three different domains can be distinguished (see Fig. 1):

- Class $I$ :

- Accelerations and decelerations are realistic and bounded.

- All properties of jams are modeled correctly.

- The jamming transition is a first order phase transition.

- The interactions are effectively long ranged.

- Class II:

- Decelerations are unbounded, leading to effectively short ranged interactions.

- Properties 1, 3, and 4 of jams are not reproduced.

- The jamming transition is no phase transition, but a crossover.

\section{- Class III:}

- Accelerations are unbounded.

- No structure formation at all.

Throughout this article only models of class $I$ will be investigated, i.e. stable jams can occur and the flow-density relation is not unique in a certain density regime (Fig. 2). The stability of the jams is directly related to the fact that the outflow from a jam is smaller than the maximal possible flow 13 .

Recently an alternative classification of stochastic traffic models with respect to two properties has been suggested [14]:

- the stability of the high-flow states.
- the stability of the outflow interface of jams.

These stability criteria were introduced to obtain a clearer characterization of traffic flow models with the focus on their stochastic properties. The Krauss-model of class $I$ exhibits stable high-flow states and a stable jam interface [14. Stability of high-flow states means that the intrinsic stochasticity of the model is not sufficient to cause a transition into the jammed regime. Therefore, the dynamics of the Krauss-models differs from the VDR-model [15, 16], for which an unstable interface was found. For the latter, the high-flow states are truly metastable, i.e. for increasing system length the probability for a transition into the jammed state becomes equal to one. In order to emphasize the difference between the nature of the high-flow states in the two models the term bistable is used in the context of the Krauss-model.

\subsection{Dynamical equations}

To derive the underlying dynamical equations, two types of motion of vehicles are considered. The first type is free motion, the second the motion of a vehicle while interaction with another vehicle takes place. Corresponding to this, two main assumptions can be made. The free motion is bounded by some maximum velocity $v_{\max }$ :

$$
v \leq v_{\max } .
$$

It is assumed that the system remains free of collisions and that a driver always chooses a velocity that does not exceed the maximum safe velocity $v_{\text {safe }}$ which guarantees the absence of collisions:

$$
v \leq v_{\text {safe }} .
$$

$v_{\text {safe }}$ is determined from the condition that the braking distance $d(v)$ needed to stop when moving with velocity $v$ satisfies

$$
d\left(v_{f}\right)+v_{f} \tau \leq d\left(v_{l}\right)+g .
$$

The quantity on the left side is the braking distance of the following car (velocity $v_{f}$ ) including a finite reaction time $\tau$. This distance has to be smaller than the braking distance of the leading car (moving with velocity $v_{l}$ ) plus the gap $g$ between the vehicles. Furthermore the model takes into account that positive and negative accelerations are bounded:

$$
-b \leq \frac{d v}{d t} \leq a, \quad \text { with } a, b>0 .
$$

It is natural to implement these restrictions using a continuous space variable, but time is discrete with timesteps $\Delta t$. From the above restrictions the dynamics of the model can be derived.

It will be assumed that, apart from random fluctuations, every vehicle moves at the highest velocity compatible with the restrictions stated above. In this way the 
model can be formulated immediately, giving

$$
\begin{aligned}
& v_{\text {safe }}(t)=v_{l}(t)+\frac{g-v_{l}(t)}{\frac{v_{f}(t)+v_{l}(t)}{2 b}+\tau}, \\
& v_{\text {des }}(t)=\min \left\{v_{\max }, v(t)+a \Delta t, v_{\text {safe }}(t)\right\}, \\
& v(t+\Delta t)=\max \left\{0, v_{\text {des }}(t)-\eta\right\}, \\
& x(t+\Delta t)=x(t)+v \Delta t .
\end{aligned}
$$

Here the gap $g=x_{l}-x_{f}-l_{\text {car }}$ is the spatial headway between the leading car at $x_{l}$ and the following car at $x_{f}$, where $l_{\text {car }}$ denotes the length of a car. $v_{l}$ and $v_{f}$ are the velocities of leading and following cars, respectively. The safe velocity $v_{\text {safe }}$ has to be determined in accordance with condition (3). $v_{\text {des }}$ is the desired velocity representing the wish to drive as fast as possible through the acceleration $v+a \Delta t$, but also respecting the conditions (11) and (2). The random perturbation $\eta>0$ has been introduced to allow for deviations from optimal driving, where $\eta=\epsilon \xi$ and $\xi$ is a random number uniformly distributed in the interval $[0,1]$.

In the following we set $\Delta t=\tau=1$. The other generic parameters used in the simulations are

$$
a=0.1, b=0.6, v_{\max }=5, \epsilon=1.0, l_{\text {car }}=1.0 .
$$

The unit of the space coordinates is the length $l_{\text {car }}$ of one car. Another parameter is the length $L$ of the system which has been choosen to be equal to 2001 (if not stated otherwise).

\subsection{Characteristics of the model}

For the parameters chosen in (6) the Krauss-model belongs to class $I$ and exhibits a bistable region with a stable high flow branch which implies a non-unique flow-density relation. Fig. 2 shows a fundamental diagram for a system corresponding to class $I$.

The existence of a bistable regime is related to the occurance of phase separation in the system. The distribution of the gaps and velocities of a system in the jammed state has two peaks [5], i.e. there are two groups of cars in the system. It separates into a macroscopic jam and a free-flow region. According to the initial conditions there exists another system state in which cars drive with velocities close to $v_{\max }$ and the distribution of gaps possesses only one peak. These states belong to the high-flow branch in the ambiguous part of the fundamental diagram.

\section{Open boundary conditions}

One of the most significant differences between systems with open and periodic boundary conditions is the car density $\rho$, which in a periodic system is a conserved quantity. Here the density and the initial conditions (in the bistable regime) determine the stationary state completely, which allows to study the density-dependence of the macroscopic parameters. In systems with open boundary conditions (OBC) one has to deal with two different tuning

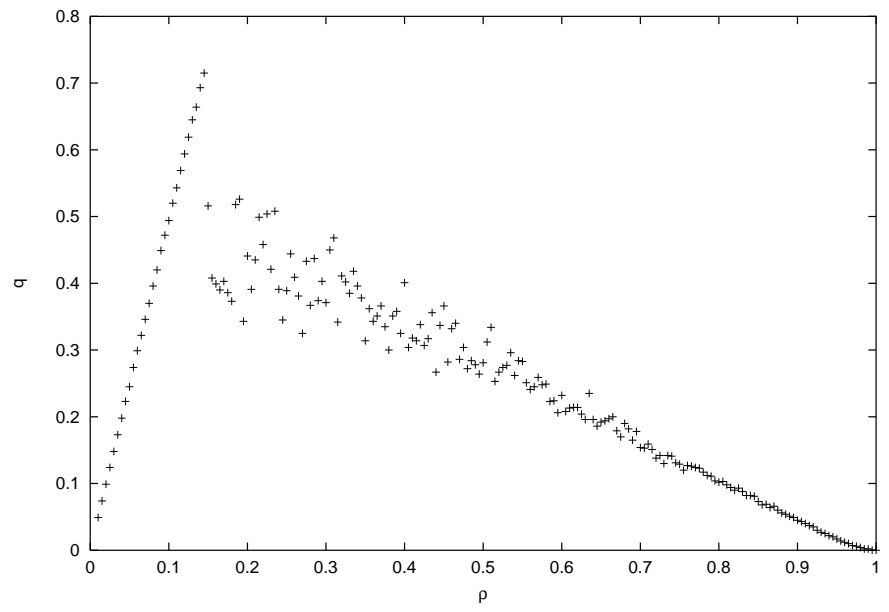

Fig. 2. Fundamental diagram of the Krauss-model with periodic boundary conditions and $a=0.1, b=0.6, v_{\max }=5$, $\epsilon=1.0, l_{\text {car }}=1.0$. The density is a mean value of densities measured in an interval of length $\frac{L}{3}$ located in the middle of a system of length $L=2001$. The flow $q$ is measured locally in the middle of the system. For densities $0.1 \leq \rho \leq 0.14$ the flow is bistable such that a stable high flow branch can be observed.

parameters, namely the injection rate $\alpha$ and the extraction rate $\beta$. So the car density in the bulk will be a result of these rates and the underlying model dynamics. In general a nontrivial density profile will develop, i.e. the average density in the system will depend on the position.

The influence of $\alpha$ and $\beta$ on the car density implies that quantities like bulk density円, current (flow) $q$, and the density profiles show a different behaviour than in periodic systems, which were studied extensively for cellular automata, optimal velocity models etc. (see e.g. [1, 2, 3] and references therein).

On the other hand, for OBC most investigations deal with simple one-component systems, especially the asymmetric simple exclusion process (ASEP) 17, 18, 19, 20, 21. In 22 the NaSch model with $v_{\max }>1$ was studied with $\mathrm{OBC}$ and the results compared to empirical data. Special boundary conditions for this case were also studied in 23 2. For driven lattice systems which exhibit a metastable or bistable regime for periodic boundary conditions not much is known about possible phase diagrams in the case of open boundaries (see, however, 16, 24), nor have systems been studied with stable high flow branches.

\subsection{ASEP with open boundary conditions}

The asymmetric simple exclusion process (ASEP) is the simplest prototype-model of interacting systems driven far from equilibrium. It is a generic model for studying driven systems and boundary-induced phase transitions [25, 26, 27 .

1 Measured by averaging over an interval of length $\frac{L}{3}$ in the middle of a system of length $L$.

${ }^{2}$ See, however, the discussion of these results in 16$]$. 
The ASEP is a discrete particle hopping model. A particle can move forward one cell with probability $p$ if the lattice site immediately in front of it is empty. If the first cell, corresponding to the left boundary ${ }^{3}$, is empty a particle will be injected there with probability $\alpha$. If the last cell is occupied the particle will be removed with probability $\beta$. By varying the tuning parameters $\alpha$ and $\beta$, and therefore the densities at the boundaries, one obtains a surprisingly rich phase diagram.

One distinguishes three different phases according to the functional dependence of the current and the corresponding stationary bulk density on the system parameters. In the low-density phase the current is independent of $\beta$. Here the current is limited by the input rate $\alpha$ which then dominates the behaviour of the system. In the highdensity phase the behaviour is dominated by the output rate $\beta$ and the current is independent of $\alpha$. In the maximum current phase the limiting factor for the current is the bulk rate $p$ and the current becomes independent of both $\alpha$ and $\beta$.

In 28] a nice physical picture has been developed which explains the structure of the phase diagram not only qualitatively, but also quantitatively. By considering the collective velocity $v_{c}=q^{\prime}(\rho)$ which is the velocity of the center of mass of a local perturbation in a homogeneous, stationary background of density $\rho$ and the shock velocity $v_{s}=\frac{q_{2}-q_{1}}{\rho_{2}-\rho_{1}}$ of a 'domain wall' between two stationary regions of densities $\rho_{1}$ and $\rho_{2}$, one can understand the phase diagram of systems with unique flow-density relation from the fundamental diagram of the periodic system 28, 29. The idea behind is that these two velocities determine if and how a perturbation will spread through the system. For a detailed discussion see 29 .

A general valid "rule" is found for systems with unique flow-density relations, i.e. the current always obeys an extremal current principle [28,29]:

$$
\begin{aligned}
& q=\max _{\rho \in\left[\rho^{+}, \rho^{-}\right]} q(\rho) \quad \text { for } \rho^{-}>\rho^{+}, \\
& q=\min _{\rho \in\left[\rho^{-}, \rho^{+}\right]} q(\rho) \quad \text { for } \rho^{-}<\rho^{+} .
\end{aligned}
$$

$\rho^{-}$and $\rho^{+}$are effective densities at the left and right boundary, respectively. The principle (7) states that the phase diagram of the open system is completely determined by the fundamental diagram $q(\rho)$ of the periodic counterpart. Moreover, it implies that two models with different microscopic dynamics, but the same fundamental diagram, will have the same phase diagram for open boundaries. In this sense the phase diagram is independent of the microscopic dynamics.

\subsection{Krauss-model with open boundary conditions}

The aim of this paper is the study of the Krauss-model with open boundary conditions, especially obtaining its phase diagram, and furthermore to investigate its connection to the theory of boundary-induced phase transitions

\footnotetext{
3 We assume that the particles move from left to right.
}

(see section 2.1). As will be shown, the choice of appropriate injection/extraction strategies at the boundaries of the system plays a crucial role. Here it should be kept in mind that the principle (7) is formulated in terms of effective boundary densities which result from these strategies.

The rules specified in the following sections have to be such that the full range of possible bulk states (compare Fig. 2) may be reached (at least theoretically). Especially, we are interested in states of high flow. Therefore, one might think of a strategy to inject cars with a initial velocity of $v_{\max }$ and injection rate $\alpha$. However, this will not lead to a crash-free motion by itself.

Since the model is known to be crash-free from the closed system, this is somehow surprising. The reason can be found from the fact that under open boundary conditions all kind of initial situations can occur due to the stochastic feeding of cars. For the safety of the model quantity $\xi(t)=g(t)-v_{l}(t)$ plays a cruicial role. Its evolution for the deterministic Krauss-model $(\epsilon=0)$ is given by

$$
\xi(t+\Delta t) \geq \xi(t)\left(1-\frac{1}{\tau_{b}+1}\right),
$$

with $\tau_{b}=\left(v+v_{l}\right) / 2 b$. Equation (8) implies that if once $\xi \geq 0$ (and as a result $g \geq v_{l} \geq 0$ ) this will hold for all future timesteps. Safety is therefore guaranteed if $\xi(t=$ $0) \geq 0$. The latter condition is not fulfilled automatically if cars are fed with a rate $\alpha$. In simulations we found that a car which collides with its predecessor always had $\xi(t=$ $0)<0$. Note that the opposite is not true, i.e. a car that started with negative $\xi$ does not have to be involved in a crash 1 . Due to the stochastic step in the update rules (5), $\xi$ can be pushed from negative to positive values but not the other way around. Just choosing smaller initial velocities reduces the probability that $\xi(t=0)<0$ leads to a crash (even to negligible values) but the states of high flow will not be reached 5 .

We follow two different strategies of injecting cars to overcome the mentioned problems. One strategy is based on the following idea: If there is enough space at the beginning of the system, i.e. at least one carlength $l_{\text {car }}$, cars are injected according to the injection rate $\alpha$. As a consequence one has to define an initial velocity $v_{\text {init }}=v_{f}(t=$ 0 ) that is as high as possible (since in the high flow states the average velocity $v \approx v_{\max }$ ), but keeps the system free of crashes. The problem here is, when using the formula for $v_{\text {safe }}$ from (5), that a velocity $v_{f}$ is already needed for its calculation. Moreover one has to deal with the cases in which $\xi(t=0) \leq 0$. Using the safety condition (3) we will derive a rule to determine $v_{\text {init }}$ in section 3 .

The other strategy investigated goes the opposite way. The high-current states of the Krauss-model are characterized by velocities close to $v_{\max }$ and more or less identical gaps $g \gg l_{\text {car }}$. This property can be used to define a

\footnotetext{
${ }^{4}$ From (8) follows that a crash is most probable for slow moving leaders and small initial gap $g_{\text {init }}$.

5 That is another difference to the NaSch-like models in which the choice of $v \leq g$ always leads to collision-free motion.
} 
rule which mimics the structure of the high current states, i.e. one defines a minimal gap $g_{\text {init }}>l_{\text {car }}$ that has to be respected at the left boundary and injects all cars with $v_{\text {init }}=v_{\max }$. It should be noted that in this case the injection rate does not equal $\alpha$ anymore, but becomes a monotoneously increasing function of $\alpha$. Details and simulation results are given in section 4 .

\section{An inflow-oriented injection rule}

In this section an injection method is introduced which is similar to that for the ASEP. Cars are injected into the system with inflow rate $\alpha$ whenever there is at least one carlength space in front of the system $\left(g_{\text {init }} \geq l_{\text {car }}\right)$, using any safe initial velocity $v_{\text {init }}$ (depending on the system configuration). However, in order to reach large currents, cars have to be injected with the maximum safe velocity possible.

The rule, as stated up to now, leads the condition $\xi(t=0)=\xi_{\text {init }}<0$ which can cause accidents as seen in section 2.2. Since we want to investigate a naive generalisation of the injection strategy of the ASEP and to compare the results to it, it is necessary to think about a rule which is mainly oriented on $\alpha$. Moreover, in realworld applications one has to understand the behaviour of such a rule, since cars usually are inserted according to a given inflow instead of particular strategies.

\subsection{Boundary rules (rule 1)}

In order to complete the rule we have to find the maximum safe velocity $v_{\text {init }}$ possible. We can not just use (3) since $v_{f}$ is not known. Moreover, the velocity has to be such that the dynamics of the system allows the transition from $\xi_{\text {init }}<0$ to $\xi \geq 0$. As a solution we do not look only at the first car in the system, but also at its predecessor.

The open boundary conditions for the inflow-oriented rule are defined in the following way:

\section{Step 1: Injection}

If there is at least one car length free space at the beginning of the system, with probability $\alpha$ we inject a car with velocity $v_{\text {init }}$ :

$$
v_{\text {init }}=\min \left\{v_{\max }, \sqrt{2 b g+\frac{b}{b_{l}} v_{l}^{2}+b^{2}}-b\right\} .
$$

This velocity is a function of $v_{\max }$, the velocity $v_{l}$ of the leading car, the deceleration rate $b$ and an upper bound $b_{l}$ for the actual deceleration of the leading car. The latter is calculated using the velocity of the car in front of the leading car and therefore, gives a bound for the worst case, i.e., the maximum deceleration of the leader in the next timestep.

Step 2: First update

Performing the first update of a car injected at the current timestep, we define an own rule. Given $v_{\text {init }}$ of step 1 we follow the update rules of the Krauss-model in case of the leading car moving with $v_{l}>v_{\text {crit }}$, where $v_{\text {crit }}$ is a constant velocity depending on $v_{\max }$. If $v_{l} \leq v_{\text {crit }}, v_{\text {safe }}$ is set equal to the gap $g$ instead of using (5). This defines a cutoff for which $\xi_{\text {init }}<0$ still leads to safe driving while keeping $g_{\text {init }}$ close to 1 for high values of $\alpha$.

Step 3: Extraction

With probability $1-\beta$ a block is added at the end of the road which causes the car at the end of the system to slow down. Otherwise, with probability $\beta$, the cars simply move out of the system.

Step 4: Update

Update with the Krauss-model update rules (see section 11).

In the following we use parameters as given in (6) and $v_{\text {crit }}=1.6, g_{\text {init }}=l_{\text {car }}=1.0$. The value for $v_{\text {crit }}$ has been determind by means of simulation. Note that $g_{\text {init }}$ is the space that has to be free at least at the left border of the system.

\subsection{Fundamental diagram}

In contrast to periodic boundary conditions, the fundamental diagram $q(\rho)$ is not easy to find for the full range of bulk densities. While for a closed system the density $\rho$ is given and conserved, in open system it is a quantity that results from the parameters $\alpha$ and $\beta$. Their influence on $\rho$ or $q$ is non-linear. Another difficulty is that global density and current should be measured in the stationary state which is reached after quite long simulation times for certain values of $(\alpha, \beta)$. A detailed discussion can be found in 30]. Because of the complex relation between $(\alpha, \beta)$ pairs and $\rho$ or $q$, one can not find a value for the $q$ for each $\rho$ and vice versa. It should be mentioned that $\rho$ and $q$ are rather sensitive to changes in $\alpha$ or $\beta$ [30].

Our strategy in finding the fundamental diagram is as follows: For each pair of parameters $(\alpha, \beta)$, the simulation has to be run until the stationary state is reached. Then, the flow $q$ and bulk density $\rho$ are measured in the middle of the system. For the latter this has been done in an interval of length $\frac{L}{3}$. These two values fix a point in the $\rho-q$ plane. To make the data more reliable and to reduce the influence of noise, this has been repeated for several times with different random seeds.

In Fig. 3 the fundamental diagram of a system with periodic boundary conditions and the same system with open boundary conditions are compared. The high-flow branch in the bistable density regime does not exist in the open case. There are also other states missing in the fundamental diagram of the system with open boundary conditions, i.e., certain densities can not be generated by the boundary rules defined in section 3.1 .

\subsection{Phase diagram}

The phase diagram as a function of $\alpha$ and $\beta$ can be obtained by studying the density profiles of systems in the stationary state. It is easy to distinguish between low- and 


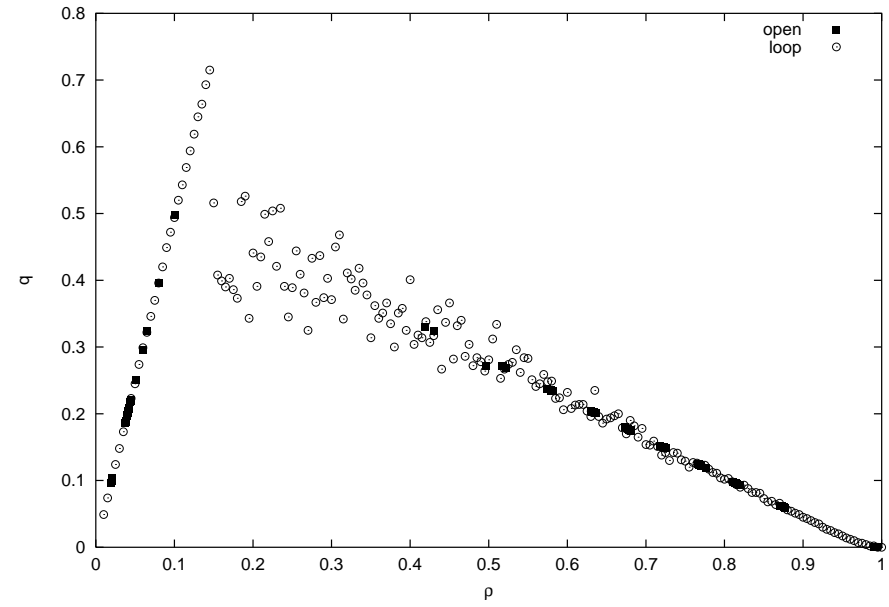

Fig. 3. Comparison between the fundamental diagram of the open system ( $\boldsymbol{\square})$ and the one from the closed system (o). The points are obtained from density profiles in case of the open system.

high-density phases. To find the maximum current phase one should compare the $(\rho, q)$ pair of the system with the fundamental diagram of the same system with periodic boundary conditions.

Using the density profiles for systems with $\alpha, \beta \in[0,1]$ with increments of 0.02 , we have drawn a phase diagram in Fig. A. Different phases, phase boundaries and the typical density profiles for each phase are shown. The maximum current phase with $q$ about 0.5 has been observed for system with open right boundary $(\beta=1)$ and injection rate between $\alpha=0.48$ and $\alpha=0.57$ (broken line). Note that all stable high-flow states in the bistable region correspond to some point in the maximal current phase.

This phase diagram looks different from phase diagrams of similar models since one finds two different low density regimes. Therefore, by varying $\alpha$ with $\beta$ kept constant, one observes a reentrance transition for large values of $\beta$. It is natural to assume that this is related to the special choice of input and output strategies. To verify this, later on (Sec. (1) a different injection strategy will be studied. The reason why this phase diagram looks unusual can be understood studying the relations between $\alpha, \beta$ and dynamic parameters of the system (see Sec. 3.4).

A similar phenomenon has been observed in 31] in a simple one-component lattice gas with next-nearest neighbour interaction. Here a reentrance transition to a second high-density phase was found. Although the origin of this transition is not entirely clear, the authors of [31] argued that it is related to the complicated connection between boundary rates and the effective boundary densities.

\subsection{Dependence of dynamic quantities on $\alpha, \beta$}

The dynamics of a system with open boundary conditions depends on the parameters $\alpha$ and $\beta$. When $\beta=1$, i.e. the outflow is unrestricted, an increasing flow can be expected with increasing injection rate $\alpha$. In Fig. 5 the flow

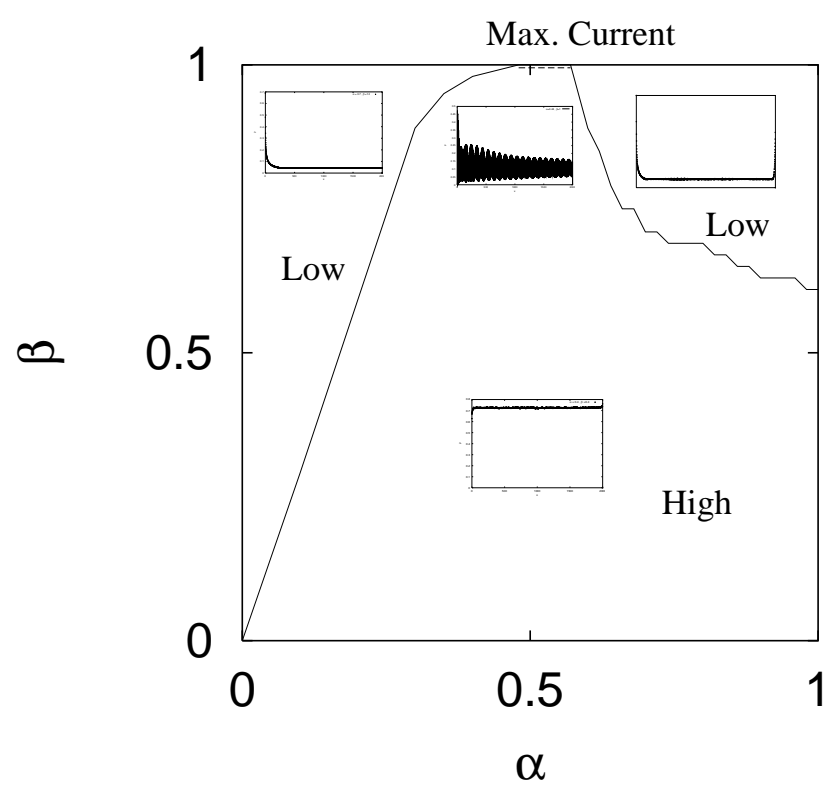

Fig. 4. Phase diagram for the Krauss-model with open boundary conditions and the inflow-oriented injection strategy. The inserts show typical density profiles for each phase. The maximum current phase with flow $q \approx 0.5$ is only observed for systems with open right boundary $(\beta=1)$ and injection rate between $\alpha=0.48$ and $\alpha=0.57$ (broken line) and shows an oscillating density profile.

of the stationary state is plotted versus $\alpha$. However, this is true only for $\alpha<0.58$. For larger values, a sharp decline in the flow can be seen which can be related to the injection strategy. Note that the second injection strategy introduced in section 1 does not show that decline in the $\alpha-q-$ relation.

For systems with $\beta \neq 1$ the right boundary introduces an external disturbance which increases with decreasing $\beta$ (cf. step 3). With $\beta \neq 1$, the cars at the end of the system are forced to slow down. Hence, the density at the right boundary increases with decreasing $\beta$ and jams are formed. These jams grow backwards into the system. If the jams can not dissolve due to high inflow rates $\alpha$, the cars have a lower average velocity and the system's density is high.

Because of the bistability, the system has quite a different behaviour for $\beta=1$ in comparison to any other $\beta$. Note that already small (external) perturbations might force the breakdown of the stable high-flow states in the open system. Once a jam has established in the system the high-flow states will not be reached again due to the reduced outflow from jams, $\left(\rho, q_{\text {out }}\right)=(0.11,0.51)$, which is smaller than the maximal possible flow. As an example for the behaviour of the system under a weak disturbance, the $\alpha-q$-relationship for $\beta=0.95$ is shown in Fig. 8 .

On the other hand, one can study the relationship between $q$ and $\beta$, using a constant value of $\alpha$ (not shown). It is obvious that for very small values of $\beta$, the current should vanish. In a system with closed right boundary 


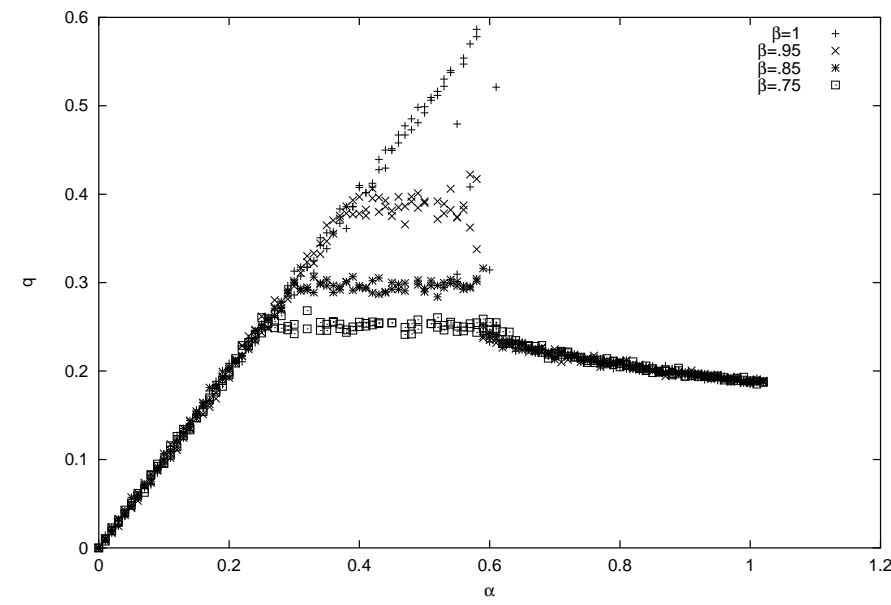

Fig. 5. $\alpha-q$-diagram for $\beta \in[0.70,1]$. The maximal reached current decreases for smaller $\beta$ since jams are builded due to the disturbance at at the right boundary of the system. The strong drop of the current for $\alpha>0.58$ is a result of the cutoff $v_{\text {crit }}$ in step 2 .

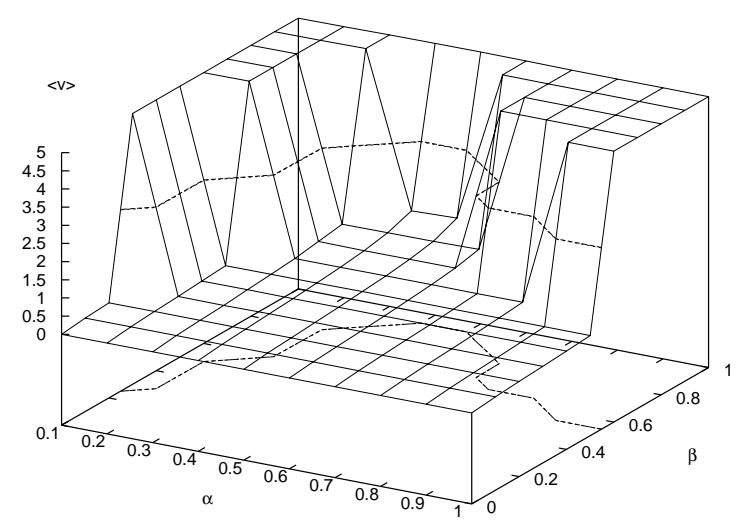

Fig. 6. Average velocity as a function of $\alpha$ and $\beta$. The contour line (- - ), given by $v>v_{\max } / 2$, separates the free flow phase (average $v$ close to $\left.v_{\max }\right)$ from the jammed phase $(v$ much smaller then $\left.v_{\max } / 2\right)$.

$(\beta=0)$, all cars are forced to stay in the system which means a vanishing average velocity $v=0$ and density $\rho=1$. For each $\alpha$, one expects the highest value of current for $\beta=1$.

In Fig. 6 the average velocity as a function of $\alpha$ and $\beta$ is shown. The contour in the $\alpha-\beta$-plane shows the line where $v=v_{\max } / 2$. In $\| 5$ this line was choosen to distinguish between jam and free flow, i.e. states with $v<v_{\max } / 2$ are in the jammed phase.

Before we will examine the connection to the extremal principle the effects of rule 1 are investigated in more detail. In order to measure the impact of that rule on flow and density we let the system run for different values of $\alpha$. In each timestep only three cars are left in the system by taking out the rest without taking care on their position

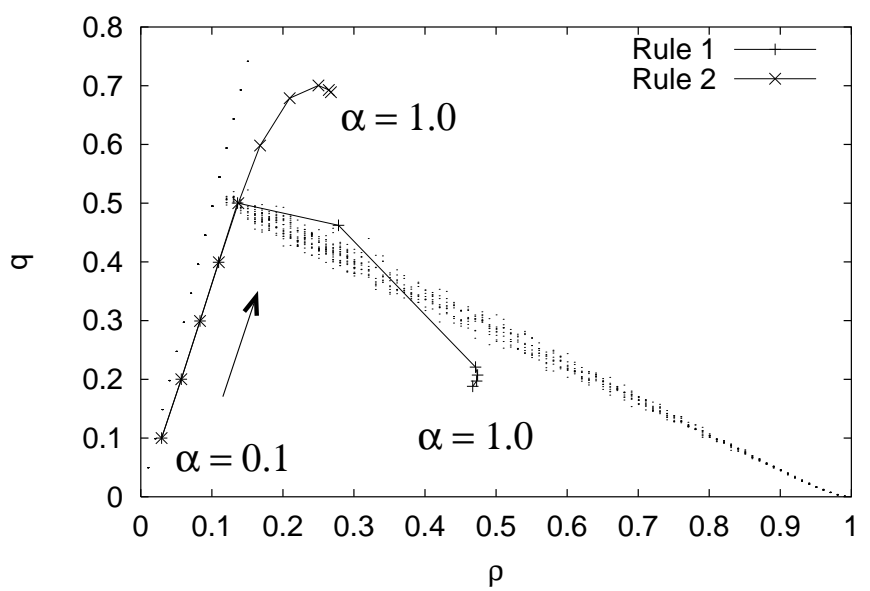

Fig. 7. Simulation of the left boundary rules given in section 3.1 (Rule 1) and 4.1 (Rule 2). In each timestep only the leftmost three cars are simulated while the rest of the system is cutted off. The dots represent the fundamental diagram taken from the closed system. After a relaxation of 100000 timesteps the points $(\rho, q)$ are measured over another 100000 steps for different values of $\alpha$. The arrow indicates the direction of increasing $\alpha$.

or velocity, i.e., the rest of the system is cutted off. The resulting relation between the density and flow at the left boundary is shown in Fig. 7. Following the line starting from $\alpha=0.1$ the left border stays on the free flow branch of the fundamental diagram up to $\alpha=0.6$. For bigger values the system switches to just one state that belongs to the jammed branch. The drop in the flow is therefore not alone a result of jams moving backwards to the left boundary, but an artificial effect of the rule itself. If the predecessing vehicles are moving slow $\left(v<v_{\text {crit }}\right)$ the safe velocity of the inserted cars becomes $g$ due to step 2 of rule 1. And, $g$ will be very small in case of high injection rates. These effect will play a role for the interpretation of the phase diagram in the context of the extremal principle.

\subsection{Extremal principle}

Since we could not observe the full range of states (cf. Fig. (3) due to the cutoff in rule 1 at a certain inflow we examined the extremal principle (see Sec. 2.1) only for a subset of system states. Since the extremal principle (7) is formulated in terms of boundary densities these have to be determinded from the stationary density profiles. For small injection rates it is not easy at all to find the left boundary density $\rho^{-}$, due to the oscillations in density profiles. This is no problem for models that have $v_{\max }=$ $l_{\text {car }}(=$ particle size $)$.

For the parts of the phase diagram that look similar to other models, i.e., all except for the second low density regime $\alpha>0.6, \beta>0.6$ (cf. Fig. 4 ), it should just be

\footnotetext{
${ }^{6}$ which results e.g. from jam formation around the left boundary
} 


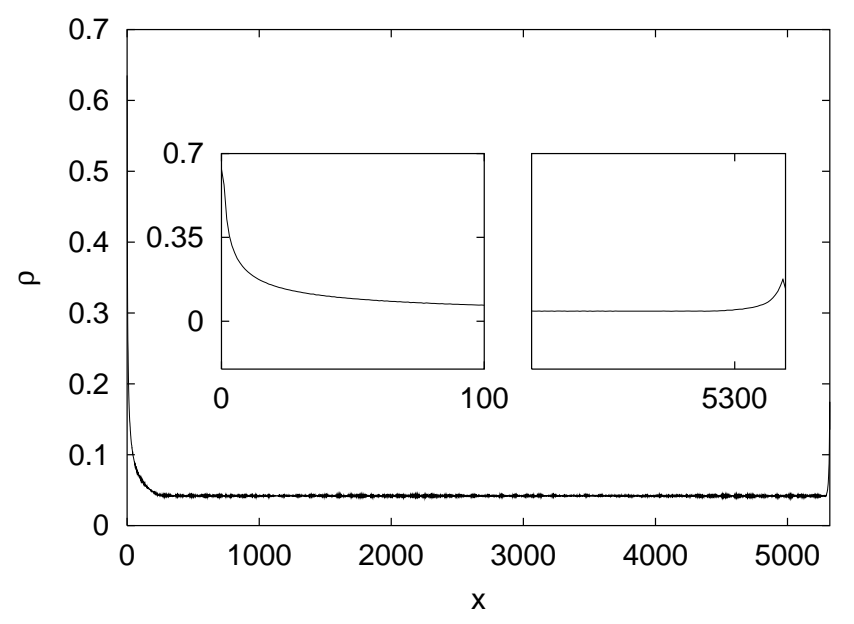

Fig. 8. Density profile for the system with $(\alpha, \beta)=(0.8,0.8)$ which lies in the second low density phase of Fig. 4. The inserts show a close up at the boundaries. The densities at the left and right boundary are $\rho^{-}=0.65$ and $\rho^{+}=0.17$ respectively, the bulk density is $\rho_{\mathrm{b}}=0.04$. A stationary flow $q=0.21$ is measured.

stated that the principle was fulfilled for all these pairs of $(\alpha, \beta)$.

A different behaviour is found for the second low density regime. The results of section 3.4 suggest that this is due to the fact that for rule 1 we do not have a monotonic increase of inflow with $\alpha$. Consider the point $(\alpha, \beta)=$ $(0.8,0.8)$ which lies in the second low density phase. Fig. 8 shows the corresponding stationary density profile. Using the formulation (7) one finds an apparent violation of the extremal principle, as will be demonstrated. From the density profiles we find $\rho^{-}=0.65$ and $\rho^{+}=0.17$ and since $\rho^{-}>\rho^{+}$the maximum of $q\left(\rho^{-}\right)$and $q\left(\rho^{+}\right)$will be chosen according to (7), i.e., $q^{\text {(pred) }}=0.48$. Instead we measure a stationary flow of $q=0.21$.

Before we present an alternative interpretation of the results in the second low density phase we recall some findings in the context of the ASEP. All the results have been obtained for models with $v_{\max }=1=\Delta t$. Therefore, in these models the flow obtruded to the system by the boundary rules does play no role. A stopped particle can accelerate to $v_{\max }=1$ in one timestep. Then, the formulation as given in (17) is sufficient to determine the system state. For models with $v_{\max }>1$ (cf. [16]) the left boundary rule has to be defined in a specific way to obtain results compatible with the extremal principle. The rule used in [16] always allows the injection of cars with $v_{\max }$. Hence large flows can be reached and the problem that acceleration to the maximum velocity takes several timesteps does not occur.

In our case the left boundary density is large due to the high injection rate $\left(\rho^{-} \propto \alpha\right)$ but the flow is restricted to $q=0.21$ due to the cutoff in step 2 of rule 1 (cf. Fig. (7). Therefore, the system has to choose a state that matches with that flow. Since the exit allows a higher flow no stable growing jam can develope in the system and the state on the free flow branch is choosen. Indeed the profile shows a sharp decrease in the density leading to a bulk density $\rho_{\mathrm{b}}=0.04$ for which $q\left(\rho_{\mathrm{b}}\right)=0.21$ (cf. Fig. 2). This indicates that the extremal principle (7) formulated only in terms of boundary densities $\rho^{-}, \rho^{+}$is not sufficient. Moreover, one has to check if there is a restriction due to the inflow and outflow rules (denoted by $q^{-/+}$). (7) then only applies for $q \leq q^{-/+}$. Otherwise the system state is chosen by

$$
q_{b}=\min \left\{q^{-}(\alpha), q^{+}(\beta)\right\}
$$

with a density $\rho_{b}$ satisfying $q\left(\rho_{b}\right)=q_{b}$. Predicting the stationary system state according to (i]) together with (10) one finds good agreement for all pairs of $(\alpha, \beta)$. The results given for another injection strategy as formulated in section 1 will confirm this interpretation. We believe that similar extensions of the extremal principle will be necessary for other multicomponent models.

\section{High-velocity-oriented injection rule}

As seen in the last section it is not easy to define left boundary conditions that allow the system to reach states belonging to the stable high-flow branch and - at the same time - ensure safe motion of the injected car under any circumstances. In the following a rule is formulated that tries to inject cars with the maximal velocity $v_{\max }$ of the model.

\subsection{Boundary rules (rule 2)}

In order to generate the high-flow states we have a closer look at the bistable regime of the corresponding periodic system. Here high-flow states are characterized by velocities close to $v_{\max }$ and approximately identical gaps $g \gg l_{\text {car }}$. Therefore, instead of driving with initial speed $v_{\text {init }}$ to achieve safety, whenever there is an empty space of one carlength $l_{\text {car }}$ in front of the system, we try to inject cars (with probability $\alpha$ ) with an initial velocity $v_{\max }$. To guarantee a system free of crashes, we have to introduce a minimum safety gap $g_{\text {init }}$ to the preceding car (cf. also the discussion in section 2.2). Therefore not all injection trials will be successful (see below).

Step 1 and step 2 of rule 1 are replaced by above strategy while the definition of the right boundary conditions is not changed. Using this strategy, it might happen that no car will be injected for several timesteps because of the lack of free space $\left(g_{\text {init }}\right)$ at the beginning of the system. This implies that the actual injection rate is smaller than $\alpha$ and also the existence of a maximal density $\frac{1}{g_{\text {init }}+1}$ which can be reached at the left boundary.

For the choice of the initial gap $g_{\text {init }}$ three criteria are formulated:

(i) $g_{\text {init }}$ should leave the system crash-free.

(ii) High currents (comparable to those found in the bistable regime of the periodic system) should be reached.

(iii) $g_{\text {init }}$ should be as small as possible. 


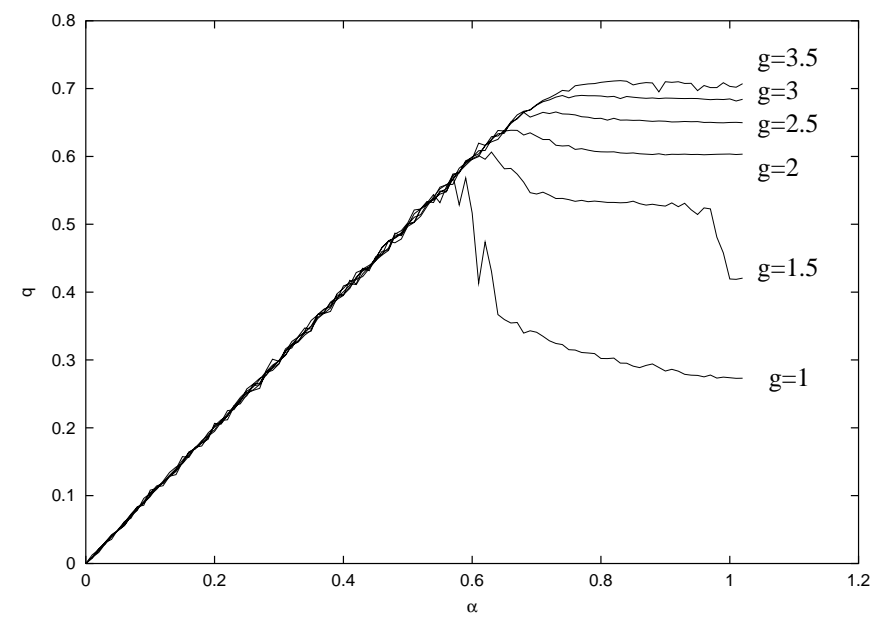

Fig. 9. The relation between the current $q$ and $\alpha$ for several values $g_{\text {init }} \in[1,3.5], \beta=1$ and the parameters equal to (6).

The last criterion increases the maximum reachable density at the system's entry so that a larger range of boundary densities can be investigated.

To find an appropriate value for the initial gap, we made several simulations with different values for $g_{\text {init }}$, and measured the current $q$ for $\beta=1$ to test criterion $(i i)$. For the values of $g_{\text {init }}$ which satisfy $(i i)$ we then checked in simulations whether the system is free of crashes. This could be done best using $\beta \approx 0$. After filtering out unsuitable values of $g_{\text {init }}$ we chose the minimum of the remaining values.

In Fig. 9 the relation between $q$ and $\alpha$ for several values of $g_{\text {init }}$ and $\beta=1$ is shown. The current increases as $\alpha$ increases and either reaches a constant value or decreases and then reaches a constant value, depending on $g_{\text {init }}$. For $5>g_{\text {init }} \geq 2$ the current reaches a constant value greater than 0.6 , which is in the bistable region of a system with periodic boundary conditions. For $g_{\text {init }} \geq 5 q$ decreases drastically. This is obvious, since it will be impossible to reach a density in the regime of maximum current in that case. The maximum current in terms of the periodic system will be reached for a system with $\beta=1$ and density of 0.15 .

In simulations for systems with $\beta=1$ and $\beta \ll 1$ we have observed crashes for $g_{\text {init }}<2$. Therefore, the only values of $g_{\text {init }}$ meeting the criteria $(i),(i i)$ are values $5>g_{\text {init }} \geq 2$. Because of the criterion $($ iii $)$ we decided to take $g_{\text {init }}=2$ in order to reach a maximum possible left boundary density.

In the following we present results of simulations using this injection rule, parameters as given in (6) and $g_{\text {init }}=$ $2, v_{\text {init }}=v_{\max }$. As long as the methods and interpretations correspond to section 3 the presentation will be kept brief.

\subsection{Fundamental diagram}

The fundamental diagram obtained for this system is very similar to the system with periodic boundary conditions. The high-flow states in the bistable regime can be reached

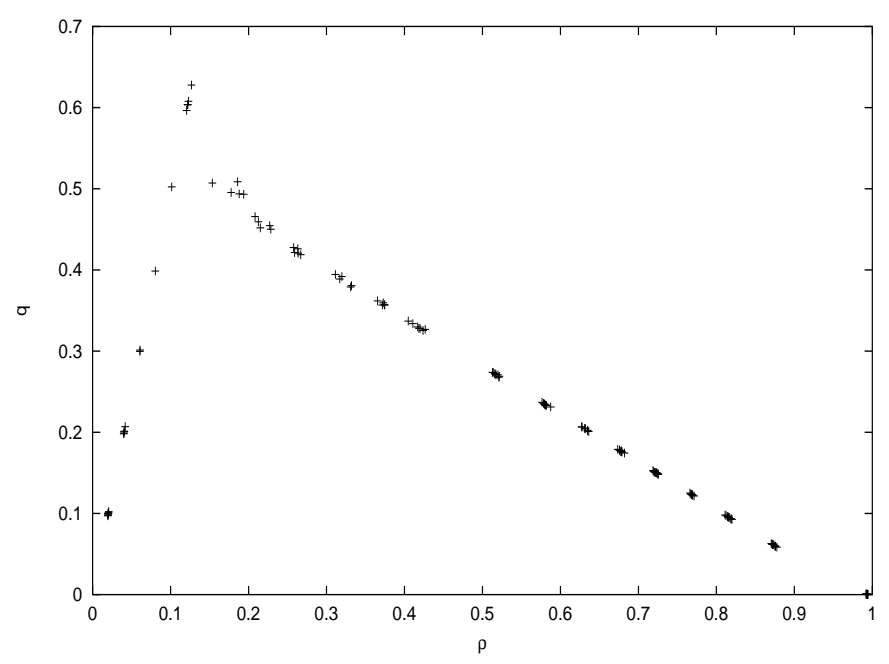

Fig. 10. Fundamental diagram of the system with open boundaries and boundary conditions defined by rule 2. The maximum current and the high-flow states in the bistable region are reached for systems with $\beta=1$. The fundamental diagram is similar to the fundamental diagram of the system with periodic boundary conditions (see section $\mathbb{1}$ ).

using rule 2, see Fig. 10. With the present injection strategy, increasing $\alpha$ does not cause the decrease of the current in the way we saw in section 3 . The high-flow states are only reached for systems with $\beta=1$ (cf. next subsections).

\subsection{Dependence of dynamic quantities on $\alpha, \beta$}

In this section we give a brief overview of the dependence of the current $q$, average velocity $v$ and the density $\rho$, measured in the middle of the system, on $(\alpha, \beta)$.

In Fig. 11 the relation between $q, \alpha$ and $\beta$ is shown. The current increases strongly for $\beta$ values close to 1 . We have also indicated the boundary between the regions with $q>0.5$ and $q<0.5$.

Fig. 12 shows the dependence of the density on $(\alpha, \beta)$. The density used here is measured in the middle of a system in the stationary state, which is the density corresponding to the plateau in the density profile for most pairs $(\alpha, \beta)$. One cleary sees a sharp transition from low to high values which will be interpreted as the phase transition line between high and low density phases.

Finally in Fig. 13 the dependence of the average velocity on $(\alpha, \beta)$ is presented. Using again the criterion $v=v_{\max } / 2$ to distinguish between free flow and jam one finds the same results for the phases as in Fig. 12.

\subsection{Phase diagram}

In Fig. 14 the phase diagram and different phases are shown. The diagram has been derived from the density profiles. The full $\alpha-\beta$-plane was scanned in steps of size 


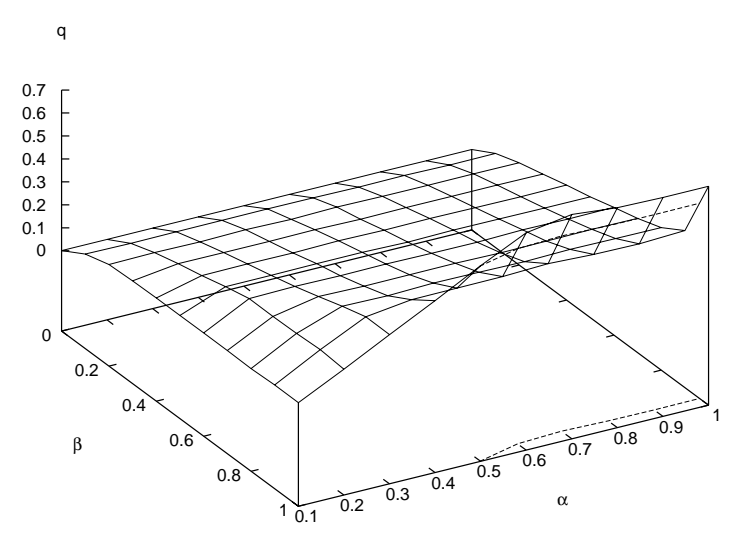

Fig. 11. The relation between the $q, \alpha$ and $\beta\left(g_{\text {init }}=2\right)$. There is no decrease of the current with increasing $\alpha$ anymore. The broken line correponds to $q=0.5$.

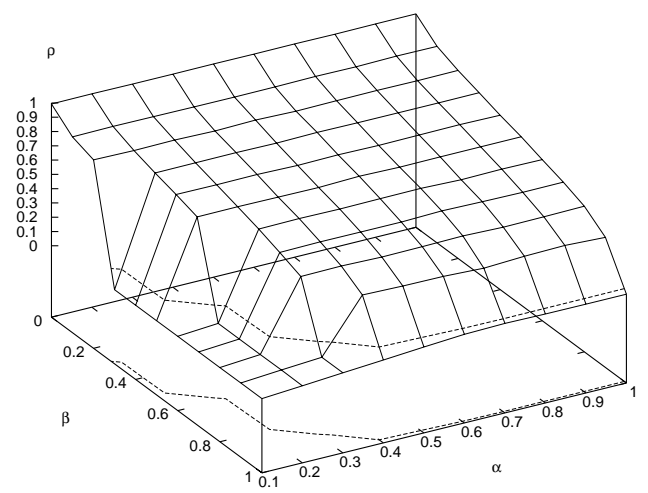

Fig. 12. The relation between the density $\rho$ (measured in the middle of the system in stationary state), $\alpha$ and $\beta\left(g_{\text {init }}=2\right)$. The broken line, corresponding to a density $\rho=0.15$, can be interpreted as a phase transition line between high and low density phases.

0.1. The maximum current phase has been observed for systems with open right boundary $(\beta=1)$. It is reached for $\alpha \geq 0.5$. As one can see from Fig. 7 at this value cars are fed in the system with the flow out of jam, i.e., $q_{\text {in }}(\alpha=0.5)=0.51=q_{\text {out }}$.

This phase diagram is similar to that of the much simpler driven lattice gas discussed in [24]. The results compare as well to the phase diagram found for the VDRmodel [16]. Therefore, we will only briefly discuss the major differences found.

For the driven lattice gas of [24] the maximum current phase is only found for very short system. The reason is the true metastable nature of the high-flow states, i.e. intrinsic fluctuations are able to destroy these states even without an external disturbance. Since the probability for

\footnotetext{
7 Using $\alpha=0.5$ and $\beta \in[0,1]$ one obtains the full high density branch of the fundamental diagram.
}

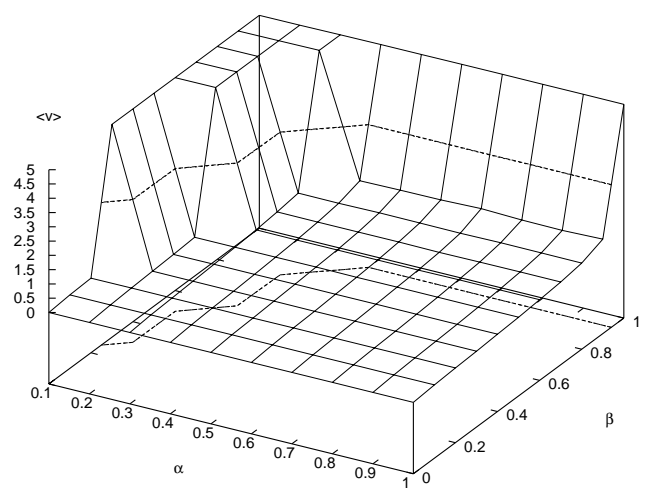

Fig. 13. The relation between the average velocity, $\alpha$ and $\beta$ $\left(g_{\text {init }}=2\right)$. The broken line corresponds to $v=v_{\max } / 2$ and can be interpreted as transition line between free flow and jammed phase.

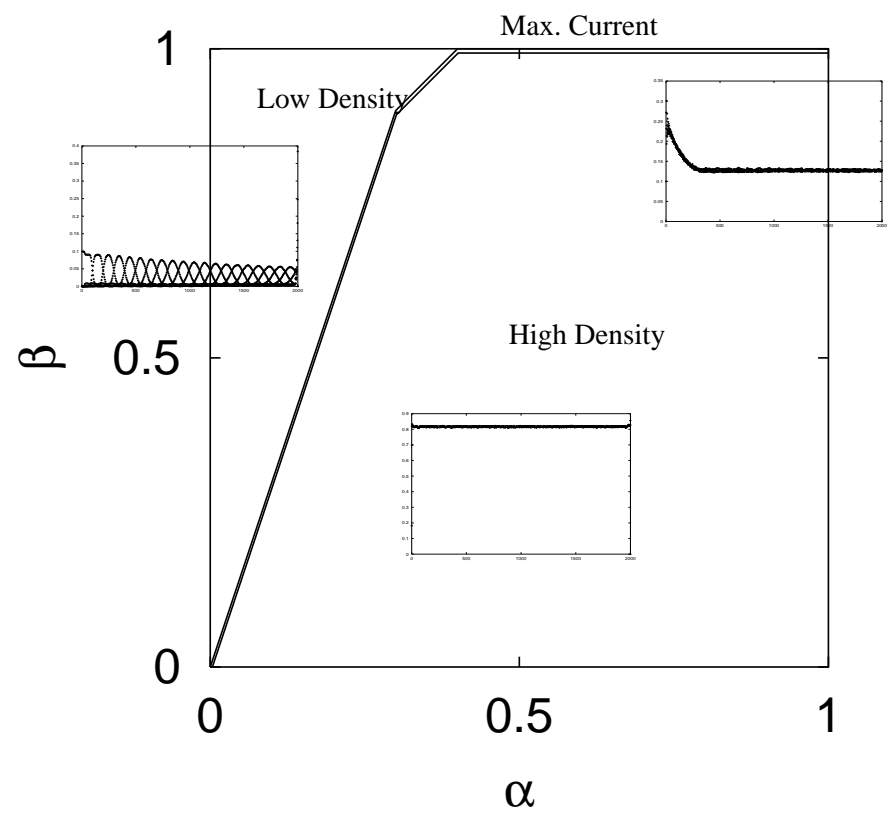

Fig. 14. Phase diagram of the Krauss-model with open boundary conditions and maximum-velocity-oriented injection rule (rule 2). The maximum current phase has been observed for systems with open right boundary $(\beta=1)$ and $\alpha \geq 0.5$. The inserts show (from left to right) typical density profiles for low, high and maximum current phase.

such fluctuations grow with the system length the maximum current phase will vanish above a typical system size. In our case, we do not find such a disappearance, even for very large systems 8 , since the high-flow states of the Krauss-model are stable (as found in [14).

The fact that we find the maximum current phase only for $\beta=1$, while in the case of the VDR-model it exists for a slightly bigger range of $\beta$, can be related to the following. The interaction in the VDR-model is very short-

8 We checked this for systems up to $L=50000$. 
ranged due to the unbounded deceleration capability in that model (the interaction horizon is $g \approx v_{\max }$ ). Instead, for the Krauss-model of the investigated class $I$, cars do already interact for $g \approx v_{\max }^{2}$.

\subsection{Extremal principle}

We examined the validity of the extremal principle for this model for every $\alpha$ and $\beta \in[0,1]$ with 0.1 steps. After measuring the boundary densities $\rho^{-}$and $\rho^{+}$we have used (7) to determine the current $q^{\text {(pred) }}$ predicted by the extremal principle. This value has then been compared to the current $q$ measured in the open system. For all parameters the results are in excellent agreement with $q^{\text {(pred) }}$. This is in accordance with our suggestion (see section 3.5) that as long as the boundary rules do not restrict the flow in an artificial way, the system will choose its bulk state according to the extremal principle as formulated in (7). We also checked our formulation with the addition given in (10). For all pairs of $(\alpha, \beta)$ the correct bulk state was predicted.

\section{Conclusions}

In this article we investigated the properties of a traffic flow model introduced by Krauss et al. [4,5,6] under open boundary conditions. It is a discrete map in time while - in contrast to cellular automata approaches space is continuous. For the parameter range discussed, the Krauss-model shows a non-unique relation between flow and density. This property of the fundamental diagram is responsible for the stability of jams found in empirical observations. It is important to note that the corresponding high-flow states have been shown to be stable 14 (subject to the model's dynamics). In contrast in cellular automata models as the VDR-model [15, 16] they usually show metastable behaviour.

For application purposes open boundary conditions play a cruicial role. But also for purely theoretical purposes the investigation of the Krauss-model's behaviour under open boundary conditions is valuable. In general, driven interacting particle systems show boundary-induced phase transitions. For systems with unique flow-density relation, e.g. the ASEP as the prototype of such models, there exists a quite general theory for the stationary state the system realized with open boundary conditions (cf. section 2). These results are well established for simple systems with unique flow-density relation [27, 28, 29]. In contrast, not much is known about for systems with a non-unique fundamental diagram. Moreover, the models investigated so far have $v_{\max }=1$ (i.e. $v_{\max }$ can be reached within one timestep). Only recently also discrete models with higher velocities (e.g. models of Nagel-Schreckenberg type 22]) and non-unique flow-density relation 16, 24 have been investigated. Here for the first time a continuous model with $v_{\max } \neq 1$ and fundamental diagram with bistability, i.e. stable high-flow states, has been studied with respect to boundary-induced phase transitions.
In order to guarantee that the system remains free of crashes, one has to find a strategy to inject cars into the system. In this article two methods are discussed. The first one is orientated on the inflow rate $\alpha$, i.e. with probability $\alpha$ a car is always inserted if there is enough space at the left boundary. In this case one has to find an initial velocity which guarantees a crash-free motion of each inserted car at any timestep (cf. section 3). The second method fixes a minimum free space $g_{\text {init }}$ at the beginning of the system $\left(g_{\text {init }}>l_{\text {car }}\right)$ and cars are injected with the constant velocity $v_{\max }$ (cf. section 4 ).

For both rules the phase diagram has been derived from computer simulations and, as expected, boundaryinduced phase transitions were found. From the ASEP three different phases are known, distinguished by the functional dependence of the current through the system on $\alpha$ and $\beta$. These are the low-density, the high-density and the maximum-curent phase. All these phases are observed in the Krauss-model with open boundary conditions.

In contrast to the findings in the ASEP 17, 18, 19, 20, 21 the maximum current phase was only observed for an open right boundary $(\beta=1)$. This reflects the high sensitivity of the Krauss-model to an external disturbance at the system's exit which results from the long interaction horizon of the model. However, in contrast to 24] the maximum current phase in the Krauss-model exists for arbitrary system lengths due to the stability of the high-flow states.

Moreover, the extremal principle (77) [28, 29 has been checked for both rules. For systems with $v_{\max }=1$ it was found that the selection of the system's state does not primarily depend on the parameters $(\alpha, \beta)$, but on the resulting densities $\rho^{-}$and $\rho^{+}$at the left and right boundary, respectively. Furthermore, the selected state is completely determined by the flow-density relation of the corresponding periodic system. In that sense the extremal principle states implies the independence of the system state from the specific injection/extraction rule at the boundaries. For the second rule we found absolut agreement for the Krauss-model (recall that here several timesteps are needed to accelerate to $\left.v_{\max }\right)$.

However, for the first rule deviations were found from the principle. An unusual reentrance transition to a second low-density phase occurs for high insertion and extraction rates. Its existence could be ascribed to the behaviour of the rule which shows a cutoff in the maximum flow for $\alpha \geq 0.6$. This flow is lower than the one that is allowed by the right boundary conditions. In other words, for a model with $v_{\max } \neq 1$ the selection of the system's state is not independent of the specific boundary rules. We therefore argue that the extremal principle as given in [28, 29] can only be applied in this strict form to models with $v_{\max }=1$. Here the provided maximum flow by the rule plays no role, since a stopped car can always accelerate to $v_{\text {max }}$ in one timestep. Therefore any flow limitation due to the insertion rule does not play a role. This is different in the case of our model which has $v_{\max }>1$ and finite acceleration capability. However, if one takes an additional 
rule into account, i.e. that the flow in the system can not exceed the flow allowed by the boundary rules, one can also predict the system state with open boundary conditions for our first rule (cf. section 3) from the knowledge about the periodic system.

We will close with a brief outlook. Due to our findings more work on boundary-induced phase transitions for models with $v_{\max }>1$ seems to be necessary. The focus should be on finding a compact formulation of the extremal principle that is independent of $v_{\max }$. Since in the Krauss-model crash-free motion is not included as a rule per se (as, through hard-core exclusion, in the cellular automata approaches) it would be helpful to develop a model formulation that does not show such safety problems under open boundary conditions. This would be important for applications in traffic flow simulation. To take into account next-nearest-neighbor interactions as suggested by the findings of section 3 seems to be promising 32.

Acknowledgments We like to thank Robert Barlovic, Kai Nagel and Gunter Schütz for useful discussions. AS thanks the DAAD for partial support and the Institute for Mathematics and Statistics at the University of Sao Paulo for its hospitality during the final stages of this work.

\section{References}

1. D. Chowdhury, L. Santen, and A. Schadschneider, Phys. Rep. 329, 199 (2000).

2. D. Helbing, Rev. Mod. Phys. 73, 1067 (2001) .

3. T. Nagatani, Rep. Prog. Phys. 65, 1331 (2002).

4. S. Krauss, P. Wagner, and C. Gawron, Phys. Rev. E 54, 3707 (1996); 55, 5597 (1997).

5. S. Krauss, PhD thesis, DLR-Forschungsbericht 98-08 (available from www.zaik.de/ paper).

6. S. Krauss, in: Traffic and Granular Flow '97, p. 269, M. Schreckenberg and D.E. Wolf (eds.), Springer (1998).

7. P.G. Gipps, Trans. Res. B, 15 B, 105-111 (1981).

8. K. Nagel and M. Schreckenberg, J. Physique I2, 2221 (1992).

9. M. Schreckenberg, A. Schadschneider, K. Nagel, and N. Ito, Phys. Rev. E 51, 2939 (1995).

10. B.S. Kerner and H. Rehborn, Phys. Rev. E53, 4275 (1996); Phys. Rev. Lett. 79, 4030 (1997).

11. B.S. Kerner, in: Traffic and Granular Flow '97, p. 239, M. Schreckenberg and D.E. Wolf (eds.), Springer (1998).

12. B.S. Kerner, Netw. Spatial Econo. 1, 35 (2001).

13. B.S. Kerner and P. Konhäuser: Phys. Rev. E 48, R2335 (1993).

14. K. Nagel, C. Kayatz, and P. Wagner, in: to appear in Traffic and Granular Flow '01, Y. Sugiyama et al. (eds.), Springer (2002), e-print cond-mat/0112116.

15. R. Barlovic, L. Santen, A. Schadschneider, and M. Schreckenberg, Eur. Phys. J. B5, 793 (1998)

16. R. Barlovic, T. Huisinga, A. Schadschneider, and M. Schreckenberg, Phys. Rev. E (in press).

17. B. Derrida, M.R. Evans, V. Hakim, and V. Pasquier, J. Phys. A26, 1493 (1993)

18. G.M. Schütz and E. Domany, J. Stat. Phys. 72, 277 (1993)
19. N. Rajewsky, L. Santen, A. Schadschneider, and M. Schreckenberg, J. Stat. Phys. 92, 151 (1998)

20. M.R. Evans, N. Rajewsky, and E.R. Speer, J. Stat. Phys. 95, 45 (1999)

21. J. de Gier, B. Nienhuis, Phys. Rev. E59, 4899 (1999)

22. V. Popkov, L. Santen, A. Schadschneider, and G.M. Schütz, J. Phys. 34, L45 (2001)

23. S. Cheybani, J. Kertesz, and M. Schreckenberg, Phys. Rev. E63, 016107 and 016108 (2001)

24. C. Appert and L. Santen, Phys. Rev. Lett. 86, 2498 (2001).

25. J. Krug, Phys. Rev. Lett. 67, 1882 (1991).

26. B. Schmittmann and R.K.P. Zia, in: Phase Transitions and Critical Phenomena, Vol. 17, C. Domb and J.L. Lebowitz (eds.), Academic Press, 1995; Phys. Rep. 301, 45 (1998).

27. G.M. Schütz, in: Phase Transitions and Critical Phenomena, Vol. 19, C. Domb and J. Lebowitz (eds.), Academic Press, 2000.

28. A.B. Kolomeisky, G.M. Schütz, E.B. Kolomeisky, and J.P. Straley, J. Phys. A 31, 6911 (1998).

29. V. Popkov and G.M. Schütz, Europhys. Lett. 48, 257 (1999).

30. A. Namazi, Diploma thesis, University of Cologne, Germany 2001. (available from www.zaik.de/AFS/theses.html).

31. T. Antal and G.M. Schütz, Phys. Rev. E62, 83 (2000)

32. N. Eissfeldt and P. Wagner, in preparation. 